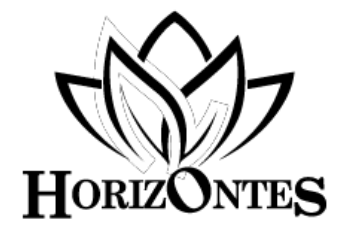

Horizontes. Revista de Investigación en Ciencias de la Educación Https: ://doi.org/10.33996/revistahorizontes.v2i5.43 enero-marzo, 2018 Volumen 2 / No. 5 pp. 38-47

www.revistahorizontes.org

\title{
Inteligencia emocional y calidad de vida en adultos mayores
}

\author{
Emotional intelligence and quality of life in older adults
}

Julliana Geraldine Paredes Carmen

caparedesju@crece.uss.edu.pe

\author{
Macarena Alicia Vidaurre Pedreros \\ vpedrerosm@crece.uss.edu.pe
}

\author{
Dina Marisol Calonge De la Piedra \\ marisolcalonge@hotmail.com \\ Universidad Señor de Sipán Chiclayo Perú
}

\section{RESUMEN}

La calidad de vida del adulto mayor es una variable compleja que depende de diversos factores, esta investigación tuvo como objetivo determinar la relación entre la inteligencia emocional y la calidad de vida en adultos mayores de una Asociación de Jubilados del Distrito de Tuman en Perú. Se utilizó un diseño no experimental, transversal correlacional. La población fue de 120 adultos mayores entre $60 \mathrm{y}$ 90 años a quienes se les administró el TMMS (Trait Meta Mood Scale) - 24 de Salovey y Mayers para medir la inteligencia emocional; y la Escala de Calidad de Vida de Olson y Barnes. La inteligencia emocional tiene tres dimensiones: Atención Emocional (AE), Claridad Emocional (CE) y Reparación Emocional (RE). La Calidad de vida se asume desde la teoría de los Dominios de Olson y Barnes con siete dimensiones o factores: 1. Hogar y Bienestar Económico (HBE), 2. Amigos, Vecindario y Comunidad, 3. Vida Familiar y Familia Extensa (VFFE), 4. Educación y Ocio (EO), 5. Medios de Comunicación (MC), 6. Religión (RE) y 7. Salud (S). Los resultados revelan que existe relación positiva entre inteligencia emocional y calidad de vida a un nivel de significancia $\mathrm{p}<0.01$. Asimismo existe relación entre claridad emocional y las dimensiones de medios de comunicación y salud $(\mathrm{p}<0.01)$; relación entre reparación emocional y las dimensiones de hogar y familia $(p<0.05)$ y medios de comunicación, religión y salud $(p<0.01)$. El nivel predominante de inteligencia emocional fue el nivel medio (59.2\%), seguido del nivel alto (40.8\%), no se encontró inteligencia emocional baja. El nivel predominante de calidad de vida es el nivel medio (49.2\%), seguido del nivel alto (31.7\%).

Palabras clave: Inteligencia emocional; calidad de vida; adulto mayo; estudio correlacional

\section{ABSTRACT}

The quality of life of the elderly is a complex variable that depends on various factors, this research aimed to determine the relationship between emotional intelligence and quality of life in older adults of an Association of Senior Citizens of the Tuman District in Peru. A non-experimental, correlational cross-sectional design was used. The population was 120 older adults between 60 and 90 years who were given the TMMS (Trait Meta Mood Scale) - 24 from Salovey and Mayers to measure emotional intelligence; and the Olson and Barnes Quality of Life Scale. Emotional intelligence has three dimensions: Emotional Attention (AE)*, Emotional Clarity (EC)* and Emotional Reparation (RE)*. The Quality of life is assumed from the theory of the Olson and Barnes Domains with seven dimensions or factors: 1. Home and Economic Welfare (HBE), 2. Friends, Neighborhood and Community (AVC) *, 3. Family Life and Extended Family (VFFE)*, 4. Education and Leisure (EO)*, 5. Media (MC)*, 6. Religion $\left(\mathrm{RE}^{*}\right)$ and 7 . Health $(\mathrm{S})^{*}$. The results reveal that there is a positive relationship between emotional intelligence and quality of life at a level of significance $p<0.01$. There is also a relationship between emotional clarity and the dimensions of media and health $(\mathrm{p}<0.01)$; relationship between emotional repair and the dimensions of home and family $(\mathrm{p}<0.05)$ and media, religion and health $(\mathrm{p}$ $<0.01$ ). The predominant level of emotional intelligence was the medium level (59.2\%), followed by the high level (40.8\%), no low emotional intelligence was found. The predominant level of quality of life is the medium level (49.2\%), followed by the high level (31.7\%).

Key words: Emotional intelligence; quality of life; older adults; correlational study 


\section{INTRODUCCIÓN}

Los conceptos de Adulto Mayor (AM) y de calidad de vida están en constante evolución y dependen de factores externos como el nivel socio económico, las características geopolíticas y culturales y de factores internos como la herencia, estado de salud, tipo de alimentación, actividad física, manejo del estrés, descanso y equilibrio emocional.

Para la Organización Mundial de la Salud - OMS - un AM es aquella persona con más de 60 años. Pérez (2013) destaca que el AM presenta dos etapas evolutivas, la primera denominada Edad Adulta Tardía, desde los 60 hasta los 75 años, llamada la edad involutiva de la experiencia. Esta etapa se caracteriza por la aceptación del envejecimiento y los cambios físicos con posibilidad de tener menos energía o padecer de alguna enfermedad. La segunda etapa, La vejez de 75 años en adelante, llamada también la edad involutiva de la sabiduría. En esta etapa se pueden presentar conflictos entre el deseo de envejecer satisfactoriamente y la ansiedad que producen los pensamientos de pérdida de la autonomía y muerte.

Gracias a las acertadas políticas de salud, a nivel mundial, la población de adultos mayores está en constante crecimiento, para el año 2050 se espera que esta población de personas mayores de 80 años alcance los 395 millones (OMS, 2016). Estas cifras indican que los Estados y las Organizaciones No Gubernamentales (ONG's) deben tener políticas para ejecutar los planes adecuados que permitan comprender y atender las necesidades que plantea el futuro para este grupo etario.

El AM no es un individuo pasivo en la relación con su entorno. Por el contrario, las decisiones que toma y sus acciones influyen en diversos grados en esta interacción. El mantenimiento de las habilidades y el derecho a elegir se encuentran estrechamente vinculados a las nociones de capacidad de acción y autonomía, que han demostrado tener gran influencia en la dignidad, la integridad, la libertad y la independencia de una persona mayor. Esto se cumple ya sea que la persona viva en la comunidad, en un centro de vida asistida o en una institución. (OMS, 2015).

Por otra parte, que en esta investigación la calidad de vida se asumirá desde la teoría de los dominios propuesta por Olson y Barnes (1982) para estos autores la calidad de vida es una percepción subjetiva que tiene la persona en relación con las posibilidades del ambiente en el cual se desenvuelve. Consideran los dominios como las expe-riencias de vida de los individuos, se refiere a los ambientes más cercanos como el familiar hasta el entorno comunitario. Son siete las dimensiones o factores que explican la calidad de vida: (1) Hogar y bienestar económico, (2) amigos, vecindario y comunidad, (3) vida familiar y familia extensa, (4) educación y ocio, (5) medios de comunicación, (6) religión y (7) salud.

La definición de calidad de vida está asociada al estado de bienestar y condicionada por aspectos objetivos, fácil-mente medibles que pueden resumirse en tener satisfechas las necesidades básicas y aspectos subjetivos que se resumen con el grado de felicidad. (OMS, 2012). La calidad de vida del AM va a depender de su propia percepción de las bondades que tiene su existencia y ésta a su vez le proporcionará un mejor bienestar.

Por lo tanto en las personas mayores el concepto de calidad de vida cobra una singular relevancia. Es importante la preocupación por vivir, no sólo más sino mejor. "Vivir más y mejor han pasado a ser las metas básicas de las políticas sociales y sanitarias de la gran mayoría de países" (Reig, 2000). 
Razón por la cual el cuidado o atención a los adultos mayores debe contemplar condiciones para su bienestar físico, psicológico y socio-ambiental. Este cuidado puede realizarse en centros especializados o en los hogares, a cargo de familiares más jóvenes.

Como consecuencia de la dinámica de vida de las familias cada vez es más frecuente que los adultos mayores vayan a un centro especializado en su cuidado y no se realice en el núcleo familiar, como es el caso de esta investigación cuyo contexto es la Asociación de Jubilados del Distrito de Tumán. Un centro de cuidado para el AM debería tener condiciones que aseguren el bienestar físico y emocional que permitan proporcionar la mejor calidad de vida posible. Es de hacer notar que la calidad de vida tiene diferentes dimensiones las cuales se configuran de manera particular y específica en cada persona; a mayor satisfacción que tenga el individuo de sus necesidades, mejor será su proceso de envejecimiento y mayor será su calidad de vida. Los adultos mayores que gozan de una buena calidad de vida muestran una mayor satisfacción existencial, (Durán, 2010).

La OMS (2015) fundamenta el funcionamiento de los centros de atención al AM en el derecho humano a la salud, refiere que:

El derecho de las personas mayores a la salud también reivindica el elemento de la aceptabilidad de los servicios, bienes y servicios de salud, de acuerdo con las normas de la ética médica y el uso de enfoques culturalmente apropiados y con perspectiva de género (p. 16).

Para evaluar la idoneidad de un centro de cuidado se debe tomar en cuenta si los servicios están adaptados a las necesidades particulares de cada AM, ya que cada persona es una singularidad y enfrenta diversas circunstancias de salud. Para la OMS esto consiste en la Estrategia Mundial de servicios de salud integrados y centrados en las personas.

El Estado debería cumplir y hacer cumplir con las políticas de protección al AM, bajo esta perspectiva un centro de cuidado debe proveer las condiciones para que sus usuarios tengan cubiertas sus necesidades primarias como alimentación, aseo, seguridad $\mathrm{y}$ confort. Asimismo debe generar el ambiente apropiado para el equilibrio psico-social, el cual puede alcanzarse a través de diversas estrategias entre ellas el desarrollo de la inteligencia emocional.

Goleman (1995) define la inteligencia emocional como la capacidad de entender las emociones de otros, comprender las nuestras y gestionar los estados emocionales propios. Precisa que las capacidades más importantes para desarrollar la inteligencia emocional son la empatía, el autocontrol, la motivación y las habilidades sociales. En este mismo orden de ideas Salovey y Mayer (1990) coinciden que la inteligencia emocional es la capacidad de controlar y regular las los sentimientos propios y ajenos y utilizarlos como guía del pensamiento y la acción. Un instrumento que se utiliza en los estudios de inteligencia emocional es el Trait Meta Mood Scale (TMMS). Este instrumento fue desarrollado por Salovey, Mayer, Goldman, Turvey y Palfai en 1995 y evalúa los aspectos intrapersonales de la Inteligencia Emocional, en concreto, las habilidades para atender, comprender y reparar los propios estados emocionales. En España en el 2004, se diseñó una versión reducida y modificada denominada TMMS-24 por Fernández-Berrocal, Extremera y Ramos (2004). Esta versión mantiene la misma estructura que el original y, con sólo 24 ítems, evalúa tres dimensiones: Atención 


\section{Emocional, Claridad Emocional y Reparación.}

La Atención Emocional es la capacidad de percibir los sentimientos propios en el mismo momento en el cual se presentan, consiste en la percepción de los estados emocionales.

La Claridad Emocional es la habilidad para Identificar y comprender los propios estados emocionales reconociendo la causa que los genera. Presenta una competencia personal que es la integración de la emoción y la razón ya que el individuo es capaz de explicar el porqué de un estado emocional, esta capacidad permite integrar la emoción con el razonamiento y consecuentemente tomar decisiones más acertadas. La empatía consiste en posicionarse emocionalmente en el lugar de la otra persona y ser consciente de qué sentimientos tiene así como el origen de ellos y las implicaciones que pudiera tener en su vida.

La emoción es un estado anímico que manifiesta una gran actividad orgánica, reflejada en los comportamientos internos y externos.

La Reparación de las emociones es la capacidad que tiene la persona de impedir los efectos negativos de esa emoción y utilizar los aspectos positivos que permiten conocer $y$ comprender la emoción con la finalidad de actuar sin perjudicarse. La reparación emocional va dirigida hacia el propio individuo y hacia los demás, por cuanto se tiene una regulación propia y otra ajena.

La Regulación Emocional Personal es la capacidad para generar respuestas emocionalmente controladas, lo que implica reconocer los sentimientos, experimentarlos y manejar esa información para generar la conducta más adecuada.

La Regulación Emocional dirigida hacia los otros está ligada con la resolución de conflictos interpersonales; consiste en la habilidad para saber escuchar, saber responder adecuadamente, establecer acuerdos, tener valores de respeto y honestidad esta regulación permite que el individuo pueda relacionarse de forma positiva con los demás.

Estudios previos reportan que los adultos mayores pueden desarrollar su inteligencia emocional como resultado de su experiencia y de la capacidad de aceptar vivir el momento "en el aquí y en el ahora". La inteligencia emocional de los adultos mayores puede explicarse por la teoría de la Selectividad Socioemocional, esta teoría propuesta por Laura Carstensen, expone que la vida se debe centrar en experiencias gratificantes y positivas en el momento presente. Consiste en la aplicación de un filtro para destacar los aspectos positivos de las vivencias esto genera vínculos de mejor calidad y una visión más optimista. Se escoge el pasar más tiempo con personas realmente significativas $\mathrm{y}$ realizando actividades gratificantes para el AM (Márquez-González, Izal, Monotorio y Losada, 2009).

La experiencia también es un factor que se considera positivo para el desarrollo de la inteligencia emocional en el AM, ya que por la cantidad de años vividos las personas tienen la posibilidad de comprender las situaciones adversas con diferentes visiones y mostrar mayor capacidad de autocontrol. Goleman (1995) señala que la vida emocional tiene un sustrato neurobiológico pero esto no es una limitante para que los individuos tengan la posibilidad de aprender a modificar de manera significativa sus respuestas modulando y modelando las mismas a través de la aplicación de técnicas o estrategias desarrolladas para conseguir la inteligencia emocional.

Los adultos mayores que se encuentran en la Asociación de Jubilados del Distrito de Tumán que constituyen la población en este 
estudio, presentan una serie de emociones negativas que los llevan a sentirse solos, desprotegidos y esto les impide tener calidad de vida y bienestar. Esta realidad no es solo de este centro sino que en forma general se visualiza la poca importancia que se le da a la atención de las necesidades de los adultos mayores; en consecuencia éstos presentan numerosas carencias en el cuidado a su salud, esparcimiento y seguridad social. Es importante realizar investigaciones que permitan comprender la realidad y sustentar propuestas efectivas para mejorar la calidad de vida del AM. El desarrollo de la inteligencia emocional tiene fundamento empírico para considerarla como una vía para conseguir mejorar la calidad de vida de los adultos mayores, por lo antes planteado esta investigación se planteó el siguiente enunciado: ¿Cuál es la relación entre la Inteligencia Emocional y la Calidad de Vida en Adultos Mayores de una Asociación de Jubilados del Distrito de Tumán entre el 2015 y 2016 ?

La investigación tuvo como propósito determinar la relación entre la Inteligencia Emocional y la Calidad de Vida en Adultos Mayores de una Asociación de Jubilados del Distrito de Tumán entre el 2015 y 2016. Para caracterizar la Inteligencia emocional, describir la calidad de vida en los adultos mayores y establecer la relación entre la Inteligencia emocional y la calidad de vida en los adultos mayores de una Asociación de jubilados.

\section{MÉTODO}

La investigación se enmarca en el paradigma cuantitativo, es del tipo confirmatorio puesto que por medio de la recopilación de datos tiene como objetivo la comprobación hipótesis. Se utiliza la medición y el análisis estadístico con el fin de establecer pautas de comportamiento y comprobar teorías (Hernández, Fernández y Baptista, 2014). El diseño es no experimental de campo, transversal y correlacional el cual permite estudiar los eventos de investigación en el contexto en el cual se desarrollan, sin manipulación de variables; los datos se recogen en un solo momento con el fin de establecer correlaciones entre dos o más variables (op. cit.). El diseño puede representarse como:<smiles>[Y]</smiles>

$\mathrm{X}_{1}$ : Variable inteligencia emocional

$\mathrm{Y}_{1}$ : Variable calidad de vida

$\Theta$ : Relación entre las variables de estudio

La población quedó conformada por 120 adultos mayores (hombres y mujeres), con una edad comprendida entre 60 y 90 años, que pertenecían a la Asociación de Jubilados del Distrito de Tumán, de la Provincia de Chiclayo en el Perú. La técnica de recolección de datos se hizo a través de la encuesta

RESULTADOS

Existe una correlación positiva débil entre las variables del estudio, la inteligencia emocional y la calidad de vida. Las variables están relacionadas ya que el nivel de significancia es $\mathrm{p}<0,01$.

Esta relación indica que mientras mayor es la capacidad de inteligencia emocional proporcionalmente la persona tiene una mejor percepción de la calidad de vida, estos hallazgos coinciden con los de Barquín (2014), quien establece que la inteligencia emocional se relaciona significativamente con la autoestima; por lo tanto, con el presente resultado y el de Barquín, se deja en evidencia que cuando un AM es capaz de procesar la información emocional, percibirla, valorarla, aprobarla, comprenderla, y regularla inteligencia emocional- (APA, 2010), también está satisfecho con su vida, además de 
sentirse emocional, física y materialmente bien, percibiendo que tiene oportunidades de desarrollo personal -calidad de vida- (APA, 2010), y percibe que sus cualidades y características son positivas, reflejando una saludable visión de lo que ha logrado y de lo que es él -autoestima- (APA, 2010), se entiende que mientras más capacidades tenga un AM este podrá controlar y regular sus sentimientos y utilizarlos como guía (Salovey y Mayer 1990), también estará más satisfecho con sus experiencias vitales como el hogar, la familia, la salud, el ocio (Olson y Barnes, 1982).

Asimismo los resultados del estudio apoyan lo planteado por Urrutia y Villarraga (2010), quienes manifiestan que los adultos mayores deben estar preparados para defender su calidad de vida, para lo cual juega un valor importante la capacidad emocional que este tenga, para ello la inteligencia emocional es un factor importante para que el
AM atienda las situaciones relacionadas con su calidad de vida, tal como mencionan, la inteligencia emocio-nal es una herramienta que promueve el logro y el afrontamiento de diversas problemáticas.

\section{Inteligencia emocional}

La inteligencia emocional de la población está entre el nivel Alto el 40,8 \% y en el nivel medio correspondiente al 59,2\%, no se evidenciaron bajos niveles de inteligencia emocional.

Para las dimensiones de la inteligencia emocional: Atención, Claridad y Reparación. Se obtuvo que la Atención emocional el 80,8 $\%$ de la población está entre el nivel medio y alto. En la dimensión Claridad emocional predomina el nivel alto con un 63,3\% del total y la Reparación emocional la mitad (50\%) de la población se ubica en el nivel alto (ver gráfico 1).

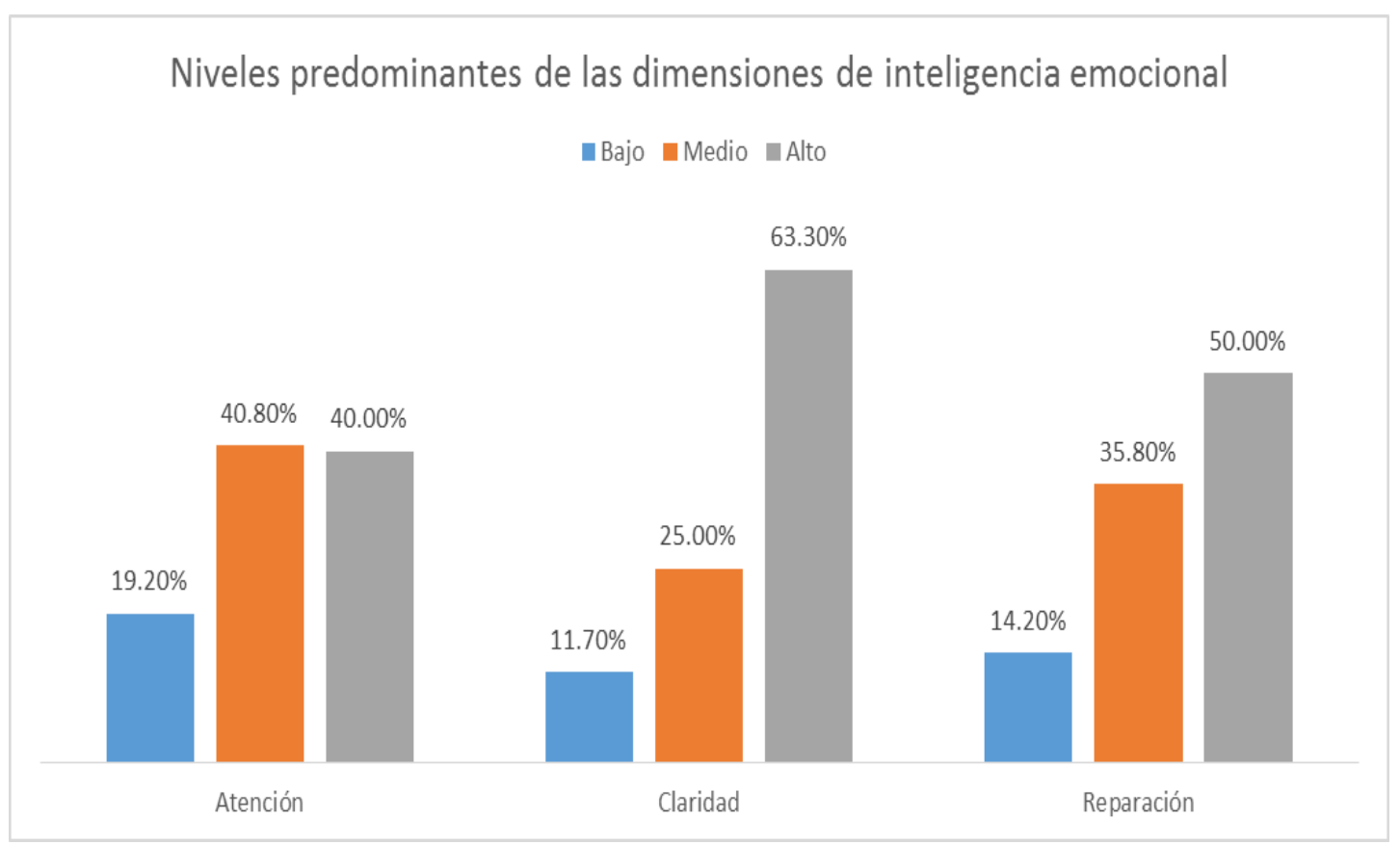

Grafico 1. Dimensiones de Inteligencia Emocional de los adultos mayores de la Asociación de Jubilados del Distrito de Tumán. 
La información arrojada en la gráfica concuerda con lo planteado por MárquezGonzález, Izal, Monotorio y Losada (2009) y López et. al. (2012) quienes sustentan que los adultos mayores tienen la capacidad de desarrollar la inteligencia emocional ya que por su experiencia de vida cuentan con las competencias suficientes como para resolver situaciones intra y extrapersonales asimismo tienen la habilidad de comprender y controlar sus propias emociones.

Sin embargo, al comparar los hallazgos con población nacional, estos se diferencian con los resultados de Ballón y Montesinos (2012), quienes encontraron niveles predominantemente bajos de coeficiente de inteligencia emocional, estas diferencias entre los resultados de Ballón y Montesinos y los del presente estudio se pueden deber a características particulares en cuanto a la atención que reciben los adultos mayores. En esta investigación la población de adultos mayores son beneficiarios de una Asociación de Jubilados, que acuden al centro de forma diurna, en cambio en la muestra de Ballón y Montesino (2012) son adultos mayores que viven en asilos, quienes están permanentemente dentro de las instituciones, por lo que esta característica puede influir en su emocionalidad, esta observación requiere de mayor comprobación y análisis.

\section{Calidad de vida}

En relación con la variable calidad de vida, los adultos mayores que conforman la población el 49,17\% declaran que tienen una calidad de vida en el nivel medio y $31,67 \%$ nivel alto. En forma general se destaca que la calidad de vida de los 120 adultos mayores están entre el nivel medio y alto. Estos resultados se aproximan a los reportados por Martínez y Pérez (2006) en su investigación sobre calidad de vida en adultos mayores reportan un $46 \%$ en el nivel medio de calidad de vida; asimismo a nivel local el estudio de Torres y Torres (2013) encontraron que en su muestra la calidad de vida es buena.

En forma específica al analizar los resultados de las dimensiones de la Calidad de Vida, solo la dimensión Hogar Bienestar Económico (HBE) se clasifica en el nivel bajo constituyendo el $49,2 \%$ de la población, lo que indica que los jubilados no se encuentran satisfechos con sus necesidades básicas (Olson y Barnes, 1982), estos resultados son congruentes con los de Acevedo, González, Trujillo, y López, (2014), quienes determinan que los adultos mayores muestran un bajo nivel de satisfacción con respecto a su bienestar económico.

Para las otras dimensiones se consiguen resultados en el nivel alto en Vida Familiar y Familia Extensa (VFF), Educación y Ocio (EO) y Salud (S). Para las dimensiones Amigos, Vecindarios Comunidad (AVC), Medios de Comunicación (MC) y Religión (RE) se ubicaron en el nivel medio (Ver gráfico 2) 


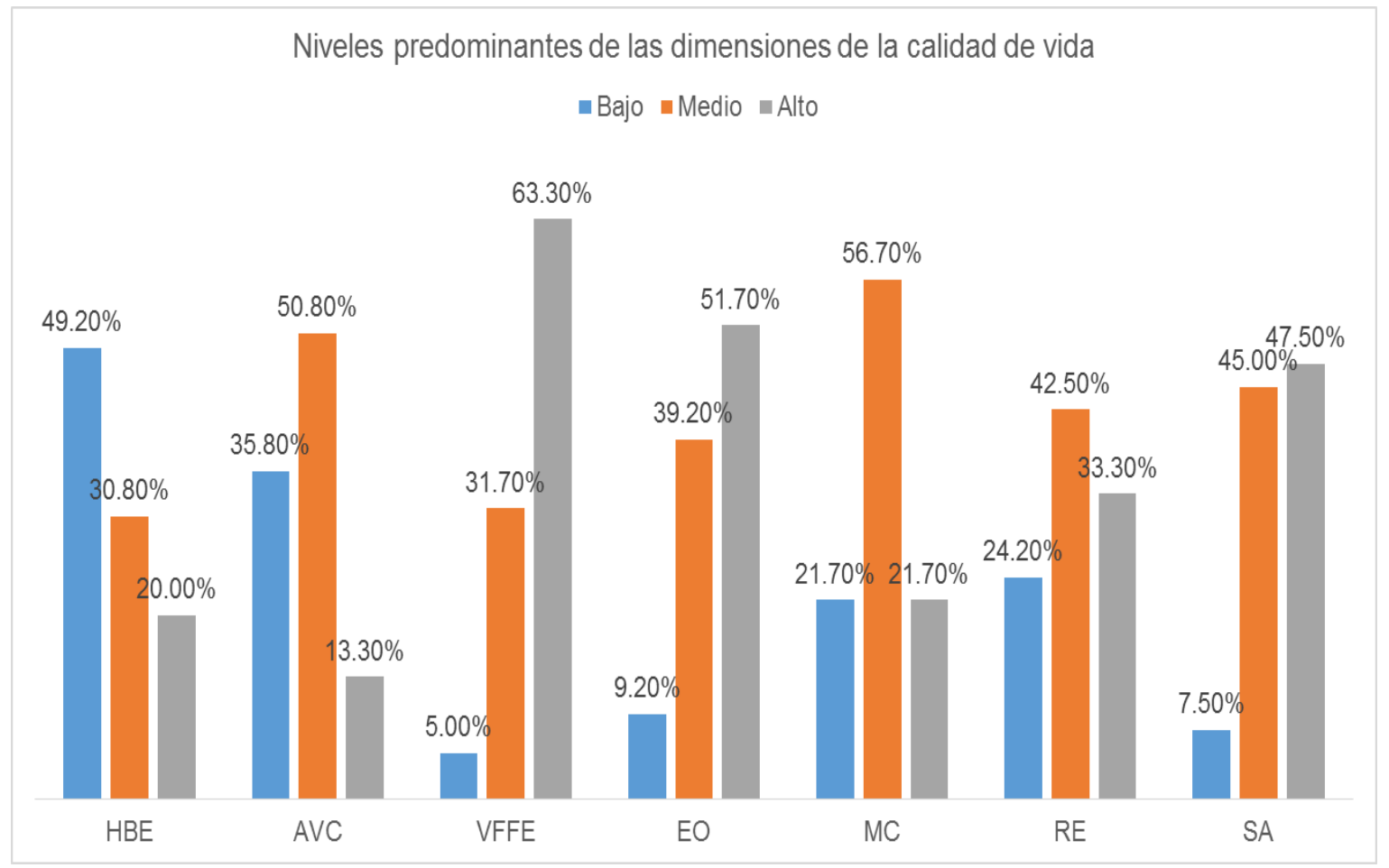

Gráfico 2. Dimensiones de la Calidad de vida de los adultos mayores de la Asociación de Jubilados del Distrito de Tumán.

\section{Relaciones entre las dimensiones de Inteligencia Emocional y Calidad de Vida}

Se establece relación entre la Atención Emocional (AE) y las dimensiones de Amigos, Vecindarios y Comunidad (AVC), Educación y Ocio (EO), Medios de Comunicación (MC), Religión (RE) y Salud (S) en adultos mayores, a un nivel de significancia. Esto se debe a que la percepción o atención emocional tiene que ver con los registros de estímulos que aprecia Salovey y Mayers en (López y otros, 2012).

En este mismo orden de ideas los hallazgos muestran la existencia de relación entre Claridad Emocional (CE) y las dimensiones de Medios de Comu-nicación (MC) y Salud (S) en adultos mayores, a un nivel de significancia.

También el presente estudio reporta que están relacionadas la Reparación Emocional (RE) y las dimensiones de Hogar y Bienestar
Económico (HBE) con Medios de Comunicación (MC), Religión(R) y Salud (S) en adultos mayores; esto nos indica diferencias en cuanto a las asociaciones. Estos resultados coinciden con los de Tapia (2014), quien encontró que las actitudes prosociales se relacionan con la calidad de vida, teniendo en cuenta que la actitud prosocial se refiere a habilidades y destrezas interpersonales, algo que teóricamente es semejante a lo que manifiesta Salovey y Mayer en López (2012), quien menciona que la reparación emocional tiene que ver con habilidades interpersonales de regulación y resolución de conflictos con los otros, por lo cual, los resultados del presente estudio, refuerzan empíricamente que la inteligencia emocional está relacionada con la calidad de vida, por lo cual es importante que se conozca la existencia de ésta relación, para que se atiendan con diferentes medios como actividades lúdicas, 
recreación, terapia ocupacional ya que estos favorecen al bienestar y calidad de vida de los adultos mayores (Urrutia y Villarraga, 2010).

\section{Hipótesis}

Para la comprobación de las hipótesis se aplicó el Coeficiente de Correlación de Pearson (r) se tienen los siguientes resultados:

\section{Hipótesis de Investigación:}

Hi. Existe relación entre inteligencia emocional y calidad de vida de adultos mayores de una Asociación de Jubilados del Distrito de Tumán.

El valor de relación es $r=0,474$ y un valor de significancia $p=0,000$; por lo tanto, se acepta la hipótesis y se concluye que existe relación positiva débil entre inteligencia emocional y calidad de vida de adultos mayores de la población en estudio, a un nivel de significancia $\mathrm{p}<0.01$ aceptándose la hipótesis con un 99\% de confianza.

\section{Hipótesis específicas}

Hi1. Existe relación entre la atención emocional y la calidad de vida de los adultos mayores de una Asociación de Jubilados del Distrito de Tumán.

Los valores del coeficiente de correlación de Pearson son: atención y amigos $(\mathrm{r}=0,474$; $\mathrm{p}=0,001)$, atención educación $(\mathrm{r}=0,252$; $\mathrm{p}=0,005)$, atención y medios de comunicación $(\mathrm{r}=0,306 ; \mathrm{p}=0,001)$, atención $\mathrm{y}$ religión $(\mathrm{r}=0,270 ; \mathrm{p}=0,003)$, atención $\mathrm{y}$ salud ( $\mathrm{r}=0,360 ; \mathrm{p}=0,000$ ); por lo tanto, se concluye que existe relación positiva débil entre atención emocional y las dimensiones de amigos, educación, medios, religión y salud en adultos mayores de una asociación de jubilados del distrito de Tumán, a un nivel de significancia $\mathrm{p}<0.01$ aceptándose la asociación con un 99\% de confianza.
Hi2. Existe relación entre la claridad emocional y la calidad de vida de los adultos mayores de una asociación de jubilados del distrito de Tumán.

El coeficiente de correlación de Pearson tomó los siguientes valores: claridad y medios de comunicación ( $\mathrm{r}=0,251 ; \mathrm{p}=0,006)$, claridad y salud ( $r=0,276 ; p=0,002)$; por lo tanto, se concluye que existe relación positiva débil entre claridad emocional y las dimen-siones de medios de comunicación y salud en adultos mayores de una Asociación de Jubilados del distrito de Tumán, a un nivel de significancia $\mathrm{p}<0.01$ aceptándose la asociación con un $99 \%$ de confianza.

Hi3. Existe relación entre la reparación emocional y la calidad de vida de los adultos mayores de una asociación de jubilados del distrito de Tumán.

Los resultados indican los siguientes valores: reparación y hogar $(r=0,204 ; p=0,026)$, reparación y familia $(r=0,187 ; p=0,041)$, reparación y medios de comunicación $(\mathrm{r}=0,306$; $\mathrm{p}=0,001)$, reparación y religión $(\mathrm{r}=0,301$; $\mathrm{p}=0,001)$, reparación y salud $(\mathrm{r}=0,368$; $\mathrm{p}=0,000$ ); por lo tanto, se concluye que existe relación positiva muy débil entre reparación emocional y las dimensiones de hogar y familia a un nivel $\mathrm{p}<0.05$; además relación positiva débil entre reparación emocional y las dimensiones de medios de comunicación, religión y salud $\mathrm{p}<0.01$; en los adultos mayores de una Asociación de jubilados del Distrito de Tumán, aceptándose la asociación con un $95 \%$ y $99 \%$ de confianza respectivamente.

\section{CONCLUSIONES}

En la población de 120 adultos mayores de la Asociación de Jubilados del Distrito de Tumán:

Existe relación positiva entre inteligen-cia emocional y calidad de vida a un nivel de significancia $\mathrm{p}<0.01$. 
El nivel predominante de inteligencia emocional es el medio y alto con el $59.17 \%$ seguido del 40.83\%. Para la Atención Emocional predomina el nivel alto y medio con el 40\% y 48\%, Claridad Emocional predomina el nivel alto (63.3\%), y la Reparación Emocional predomina el nivel alto $(50 \%)$

El nivel predominante de la calidad de vida fue medio (49.17\%). En las dimensiones el nivel predominante en Hogar y Bienestar Económico (HBE) es el nivel bajo; Las otras dimensiones ubican sus resultados en el nivel alto.

Existe relación positiva débil entre Atención Emocional y las dimensiones de Calidad de Vida AVC, EO, MC, RE y S.

Existe relación positiva débil entre Claridad Emocional y las dimensiones de Calidad de Vida MC y S.

Existe relación positiva muy débil entre Reparación Emocional y las dimensiones de calidad de vida de HBE y VFFE a un nivel $\mathrm{p}<0.05$

Existe una relación positiva débil entre Regulación y las dimensiones de Calidad de Vida de MC, RE y S.

Las relaciones entre la inteligencia emocional y la calidad de vida establecidas en esta investigación deben ser consideradas para desarrollar planes de atención al AM de tal manera de incrementar los niveles de satisfacción y bienestar de los adultos mayores en el país. Se recomienda ejecutar planes que permitan el desarrollo de la inteligencia emocional a nivel formal $e$ informal.

\section{REFERENCIAS}

Acevedo Alemán, J., González Tovar, J., Trujillo Pérez, M., y López Saucedo, M. (2014). El adulto mayor usuario de centros del Instituto Nacional de las Personas Adultas Mayores (Inapam): percepciones sobre su calidad de vida en Saltillo, Coahuila, México. Perspectivas sociales, 16(1), 73-97.

American Psychological Association APA (2010). Diccionario conciso de Psicología. México: Editorial El Manual Moderno

Ballón Díaz, M. L. y Montesinos Deza, L. C. (2012) Inteligencia Emocional en Adultos Mayores de Asilos Públicos E Instituciones Privadas de Arequipa. Revista Psicológica Arequipa, 2(1), pp.4963. Disponible: http://goo.gl/In1mLE [Consulta: 2015, Noviembre 21]

Barquín Cruza, A. R. (2014). Relación entre inteligencia emocional y autoestima en un grupo de trabajadores en proceso de jubilación pertenecientes a una institución bancaria. Trabajo de grado, no publicado. Universidad Rafael Landívar. Disponible; http://goo.gl/jDyHAr [Consulta: 2015 Septiembre18]

Durán, M. M. (2010). Bienestar psicológico: el estrés y la calidad de vida en el contexto 